\title{
Does prophylactic ureteral catheter placement offer any advantage for laparoscopic gynecological surgery? A urologist' perspective from a systematic review and meta-analysis
}

\author{
Dechao Feng", Yin Tang", Yubo Yang, Xin Wei, Ping Han, Wuran Wei \\ Department of Urology, Institute of Urology, West China Hospital, Sichuan University, Chengdu, China \\ Contributions: (I) Conception and design: D Feng; (II) Administrative support: P Han, W Wei; (III) Provision of study materials or patients: D \\ Feng, Y Tang, Y Yang; (IV) Collection and assembly of data: D Feng, Y Yang, X Wei; (V) Data analysis and interpretation: D Feng, Y Tang; (VI) \\ Manuscript writing: All authors; (VII) Final approval of manuscript: All authors. \\ "These authors contributed equally to this work. \\ Correspondence to: Wuran Wei, PhD. Department of Urology, Institute of Urology, West China Hospital, Sichuan University, Guoxue Xiang \#37, \\ Chengdu 610041, Sichuan, China. Email: weiwuranwch@126.com.
}

\begin{abstract}
Our aim is to assess the efficacy of prophylactic ureteral catheter placement on patients undergoing laparoscopic gynecological surgery. We searched the electronic database including PubMed, Cochrane Library, Embase, Web of Science, WANFANG and CNKI in January 2020 to identify possible studies without languages limitations. A manual search was also conducted. The trials that compared catheterized group (CG) to non-catheterized group (NCG) were included. This meta-analysis was accomplished by RevMan5 (version 5.3). Initial search yield 997 studies and 5 randomized control trials were included in the final meta-analysis. Pooling data of five studies showed that patients in the CG had a lower risk of ureteral injury than those in NCG (RR: 0.44, 95\% CI: 0.20-0.97, P=0.04) without significant between-study heterogeneity $\left(\mathrm{P}=0.23, \mathrm{I}^{2}=29 \%\right)$. The pooled data analysis showed a statistically significant difference in favor of prophylactic ureter catheter placement (MD: $-40.51,95 \%$ CI: -58.65 to -22.36 , $\mathrm{P}<0.0001)$. Random-effects model meta-analysis found that patients in the CG experienced higher EBL compared to NCG (SMD: $-5.78,95 \% \mathrm{CI}:-10.51$ to $-1.04, \mathrm{P}=0.02$ ). There was no statistically significant difference between CG group and NCG group ( $\mathrm{P}=0.23)$ with regard to LOS. Current evidence indicates that prophylactic ureteral catheter placement has the advantages of reducing ureteral injury, shortening the operative time, and reducing the amount of bleeding. It might serve as a routine preoperative preparation choice for laparoscopic gynecological surgery, especially with pelvic adhesion. Further large volume, multicenter well-designed trials are warranted before making the final clinical guidelines.
\end{abstract}

Keywords: Ureteral catheter; ureteral stent; double-J stent; gynecological surgery; iatrogenic ureteral injury; randomized control trial (RCT)

Submitted Feb 28, 2020. Accepted for publication Aug 14, 2020.

doi: $10.21037 /$ tau-20-674

View this article at: http://dx.doi.org/10.21037/tau-20-674

\section{Introduction}

Iatrogenic urinary tract injuries are frequent in obstetrical and gynecologic surgery owing to the proximity of the urogenital organ systems, and delayed diagnosis and inappropriate medical interventions lead to high morbidity and even mortality (1). Ureteral injury is one of the most devastating and troublesome complications in gynecologic surgery (2). Moreover, iatrogenic ureteric injuries (IUI) have increased markedly during the past two decades (3) due to advances in endoscopic gynecologic surgery during 
the late 1980s and early 1990s (2-5). Thus, how to find ureteric injury promptly and effectively reduce ureteral injury should be emphasized in the management of laparoscopic gynecological surgery. The ureteral catheter placement is a simple procedure which contributes to identifying the ureteral course. However, whether ureteral stent should be placed routinely before laparoscopic gynecological surgery remains controversial. Therefore, we conducted a systematic literature review and meta-analysis of randomized control trials (RCTs) at home and abroad to explore this relationship. We present the following article in accordance with the PRISMA reporting checklist (available at http://dx.doi.org/10.21037/tau-20-674).

\section{Methods}

\section{Study selection}

A systematic literature search in the PubMed, Cochrane Library, Embase, Web of Science, WANFANG and CNKI databases was conducted to identify RCTs published until January 2020 in accordance with PRISMA guideline (6). The language was unrestricted. Various keywords or Mesh terms including obstetric, gynecologic, laparoscopic, ureteral stent, ureteral catheter, and double-J stent, were used. The search strategy used in PubMed was as follows: (((()obstetric[Title/Abstract]) OR gynaecologic[Title/ Abstract]) OR Gynecological[Title/Abstract]) OR laparoscopic[Title/Abstract])) AND ((((double-pigtail stent[Title/Abstract]) OR Ureteral stent[Title/Abstract]) OR ureteral catheter[Title/Abstract]) OR Double-J stent[Title/Abstract]). Reference lists of all related studies were also retrieved to ensure literature saturation. All RCTs reporting the outcomes of interest were included.

\section{Selection criteria}

Two independent reviewers (D Feng, Y Tang) screened studies based on titles and abstracts. Full articles were further retrieved for possible qualified researches. Disagreement was resolved by consensus and by discussion with a third party. We defined study eligibility using the following PICOS approach. Patients $(\mathrm{P})$ : patients undergoing laparoscopic gynecological surgery; Intervention (I): prophylactic ureteral catheterization; Comparison (C): publications reporting outcomes of laparoscopic gynecologic surgery with or without preset ureter catheter; Outcomes $(\mathrm{O})$ : the following outcomes were assessed: ureteral injury; operative time; estimated blood loss (EBL) and length of stay (LOS). Study design (S): RCTs. Exclusion criteria included: (I) not meeting inclusion criteria; (II) studies with overlapping or insufficient data; (III) reviews, lectures, meeting abstracts and meta-analysis.

\section{Quality assessment}

The methodological quality of the studies (Figure 1) was assessed by two independent authors (D Feng, Y Tang) using the Cochrane Collaboration's Risk of Bias (RoB) tool in Review Manager software (https://community.cochrane. org/help/tools-and-software/revman-5). This tool primarily includes 7 domains: random sequence generation (selection bias); allocation concealment (selection bias); blinding of participants and personnel (performance bias); blinding of outcome assessment (detection bias); incomplete outcome data (attrition bias); selective reporting (reporting bias); other bias (such as funding sources).

D Feng and Y Tang independently rated the level of evidence of the included articles using the Oxford Centre for Evidence-Based Medicine criteria (7); This scale graded studies from strongest (level 1) to weakest (level 5) strength of evidence on the basis of study design and data quality.

Figure 1 details the RoB summary of the 5 RCTs (8-12) included in this meta-analysis. In summary, included studies had a low risk of performance bias (blinding of participants and personnel), attrition bias (incomplete outcome data) and reporting bias (selective reporting). The risk of selection bias and detection bias were unclear due to lack of related description.

\section{Statistical analysis}

Comparable data from each study were merged in a meta-analysis where possible. We calculated pooled estimates of the mean difference (MD) or standard mean differences (SMD) and relative risk (RR) for continuous and dichotomous variables, respectively. We used the Cochran $\mathrm{Q}$ test to assess between-study heterogeneity (13). We also $\mathrm{did}^{2}$ testing to evaluate the magnitude of the heterogeneity with values $>50 \%$ regarded as being significant heterogeneity (14). The random effects model was used when the trials yielded heterogeneous $(\mathrm{P}<0.1)$ results. Otherwise, the fixed effects model was used. Significance was set at $\mathrm{P}<0.05$. The present meta-analysis was performed 


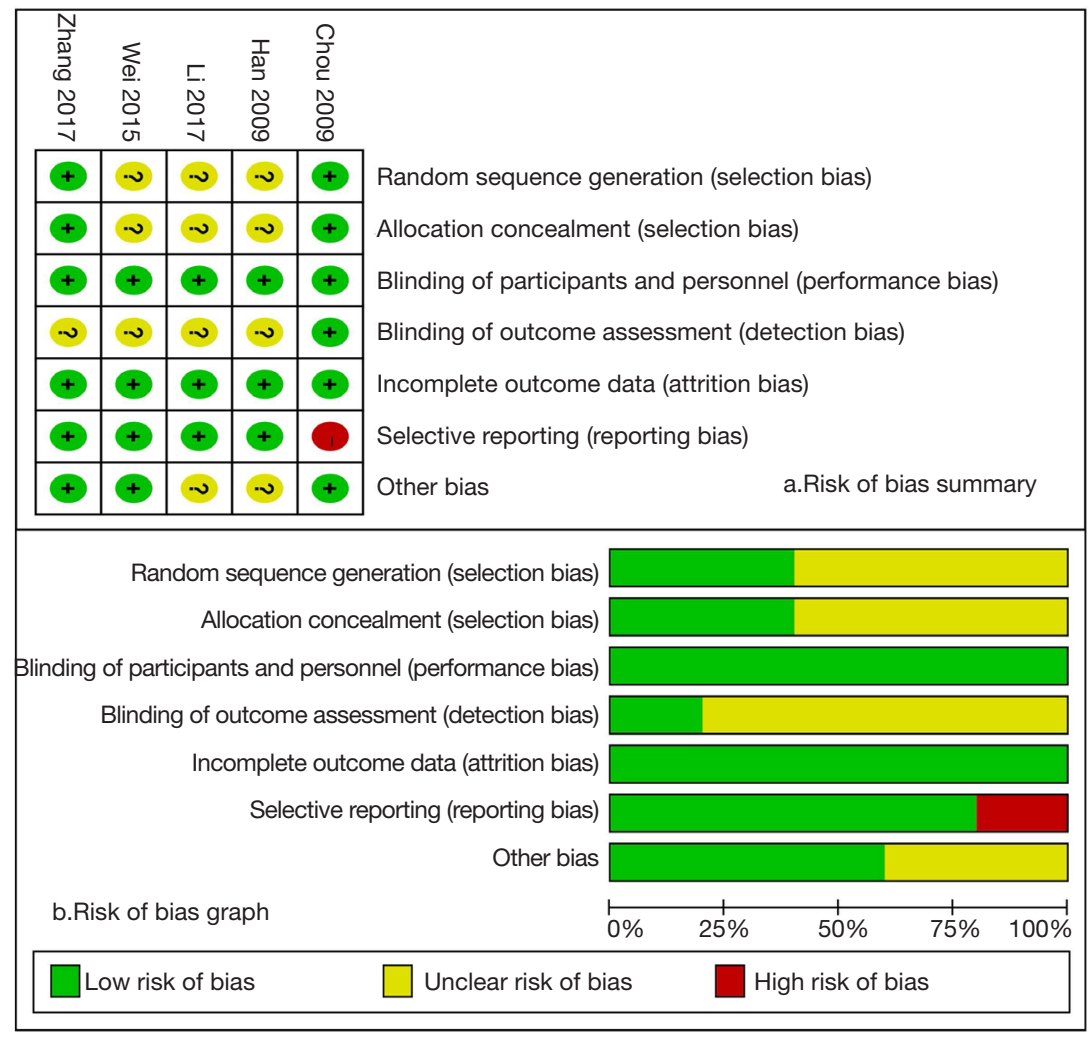

Figure 1 The risk of bias summary of included studies.

using the Cochrane software Review Manager version 5.3.

\section{Results}

\section{Literature search results}

Initial literature search yield 997 studies. After removing duplication, screening studies based on titles and abstracts and reviewing full articles, a total of 5 RCTs (8-12) consisting of 1,290 patients [676 in the catheterized group (CG) and 614 in the non-catheterized group (NCG)] were enrolled in the final analysis. The flow chart of the study selection is shown in Figure 2.

All studies were RCTs and contained patients treated with laparoscopic gynecological surgery. All 676 patients were treated with ureteral catheter placement while the remaining 614 patients did not. Two studies $(8,9)$ were published in English and three trials (10-12) were published in Chinese. All of these studies reported outcomes of laparoscopic gynecologic surgery with or without prophylactic ureter catheter placement and were carried out in China. The sample size of included studies was relatively small and only 1 article contained more than 200 patients (8). Two studies had a duration of more than 5 years $(8,9)$. One study enrolled patients undergoing laparotomy and laparoscopic gynecologic surgery (8), and we extracted the results of ureteric injury in laparoscopic-assisted vaginal hysterectomy and oophorectomy. All studies had major gynecological procedures and did not report major catheter-related complications. Table 1 details the main characteristics of included studies.

\section{Ureteral injury}

All RCTs (8-12) included in this meta-analysis reported the results of ureteral injury. Pooling data of these five studies 


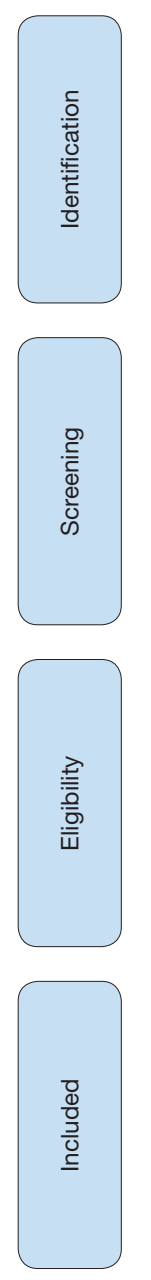

Records identified through database searching English $(n=585)$, Chinese $(n=402)$
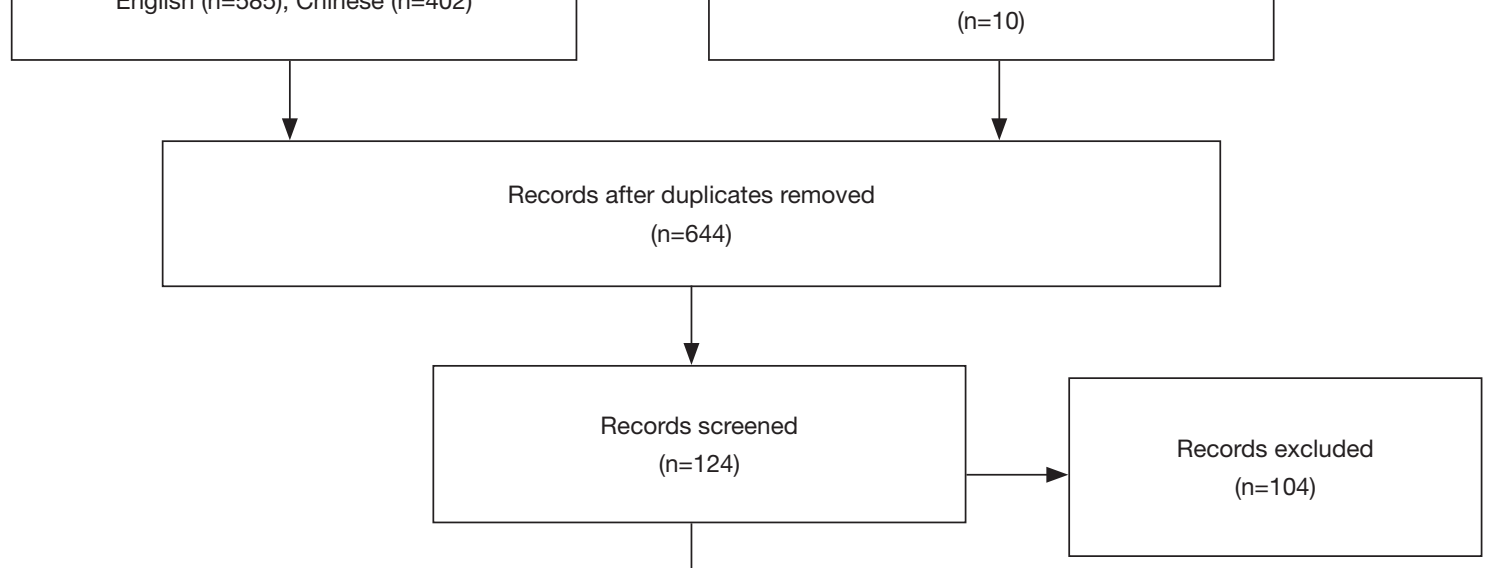

Figure 2 The flow chart of the study selection.

showed that patients in the CG had a lower risk of ureteral injury than those in NCG (RR: 0.44, 95\% CI: 0.20-0.97, $\mathrm{P}=0.04)$ without significant between-study heterogeneity $\left(\mathrm{P}=0.23, \mathrm{I}^{2}=29 \%\right)$ (Figure 3).

\section{Operative time}

Four studies (9-12) (289 patients) were included in the meta-analysis. The pooled data analysis showed a statistically significant difference in favor of prophylactic ureter catheter placement. A test for heterogeneity between the four studies was positive $\left(\mathrm{P}<0.00001, \mathrm{I}^{2}=92 \%\right)$, so a random-effect meta-analysis was conducted between the two groups (MD: $-40.51,95 \%$ CI: -58.65 to -22.36 , $\mathrm{P}<0.0001$ ) (Figure 3). We conducted sensitivity analysis by removing each study in sequence, and we did not observe a significant change after removing each trial.

\section{$E B L$}

Data from three trials $(9,11,12)$ with 264 patients were pooled. Random-effects model meta-analysis found that patients in the CG experienced higher EBL compared to NCG (SMD: $-5.78,95 \%$ CI: -10.51 to $-1.04, \mathrm{P}=0.02$ ) with highly between-study heterogeneity $\left(\mathrm{P}<0.00001, \mathrm{I}^{2}=99 \%\right)$ (Figure 3). The relatively small sample size made us unable to draw a robust conclusion with regard to EBL.

\section{LOS}

The pooled data from two studies $(9,11)$ showed no statistically significant difference between CG group and 
Table 1 The baseline characteristics of included studies

\begin{tabular}{|c|c|c|c|c|c|c|c|c|}
\hline Study ID & Country & Language & Duration & Populations & $\begin{array}{l}\text { Sample size } \\
\text { (CG/NCG) }\end{array}$ & Age, tears (CG/NCG) & Outcomes $^{\&}$ & LoE \\
\hline Chou, 2009 & China & English & $\begin{array}{l}1996.1 \text { to } \\
2007.12\end{array}$ & $\begin{array}{l}\text { Inclusion: laparoscopic } \\
\text { hysterectomy; oophorectomy. } \\
\text { Exclusion: without consent form; } \\
\text { operative time less than } 30 \text { min; } \\
\text { follow-up less than } 6 \text { months }\end{array}$ & $527 / 472$ & $41.79 \pm 12.43 / 41.98 \pm 11.56$ & 1 & $1 b$ \\
\hline Li, 2017 & China & Chinese & $\begin{array}{l}2012.5 \text { to } \\
2014.5\end{array}$ & $\begin{array}{l}\text { Inclusion: radical hysterectomy; } \\
\text { myomectomy; ovarian cyst } \\
\text { ablation }\end{array}$ & $42 / 42$ & $42.5 \pm 3.4 / 43.5 \pm 3.6$ & $1,2,3$ & $2 b$ \\
\hline Wei, 2015 & China & Chinese & $\begin{array}{l}2013.1 \text { to } \\
2015.1\end{array}$ & $\begin{array}{l}\text { Inclusion: laparoscopic } \\
\text { hysterectomy; } \\
\text { oophorectomy }\end{array}$ & $60 / 60$ & Similar group & $1,2,3,4$ & $2 b$ \\
\hline
\end{tabular}

\&, 1: ureteral injury; 2: operative time; 3: estimated blood loss; 4: length of stay. IFGO, International Federation of Gynaecology and Obstetrics criteria; LoE, level of evidence; CG, catheterization group; NCG, non-catheterization group.

NCG group (MD: $-1.20,95 \% \mathrm{CI}:-3.16$ to $0.76, \mathrm{P}=0.23$ ) with highly between-study heterogeneity $(\mathrm{P}<0.00001$, $\mathrm{I}^{2}=95 \%$ ) (Figure 3).

\section{Discussion}

With the increasing application of laparoscopy in gynecological surgery since the late 1980s, the number of IUI has increased markedly during the past two decades, partly because of the introduction of laparoscopy and the overall increase in surgical procedures $(2-3,15)$. Additionally, the incidence of ureteral injury is related to the types of operations performed at different periods and the surgeons' experience (16). The probability of ureteral injury in laparoscopic operations increases by $0.2-6.0 \%(2,17)$. Most doctors who can perform a radical hysterectomy have experience with laparotomy and basic laparoscopic surgery. However, due to insufficient understanding of the anatomy around the cervix under the microscope, unskilled manipulation, and the use of electric energy devices, urinary tract injuries are prone to occur in the early stages of such surgery (17). Among these complications, IUI is one of the most serious complications (2). Furthermore, laparoscopic radical hysterectomy requires opening the ureteral tunnel, which also increases the chances of ureteral injury and other severe complications (17). Notably, although laparoscopic surgery has a higher risk of ureteral injury than laparotomy, patients prefer laparoscopic surgery over open surgery due to less scarring, shorter LOS and less postoperative pain $(2,17)$. IUI not only severely affects the physical and mental health of patients, but also increases the risk of doctorpatient disputes. Thus, an ability to discriminate and avoid the occurrence of ureteral injury is within the purview of the obstetrician-gynecologist accurately and promptly.

A meta-analysis of 79 mostly retrospective studies (18) shows that routine cystoscopy clearly increases the intraoperative detection rate of urinary tract injuries with an adjusted IUI rate of $0.3 \%$ and a bladder injury rate of $0.8 \%$. How to effectively prevent the occurrence of ureteral injury is a problem that urgently needs to be solved in laparoscopic gynecological surgery. Currently, the efficacy and safety of prophylactic ureteral catheter placement before gynecological surgery is still controversial. Some experts supported the use of preoperative ureteral stent 


\section{a.Ureteral injury}

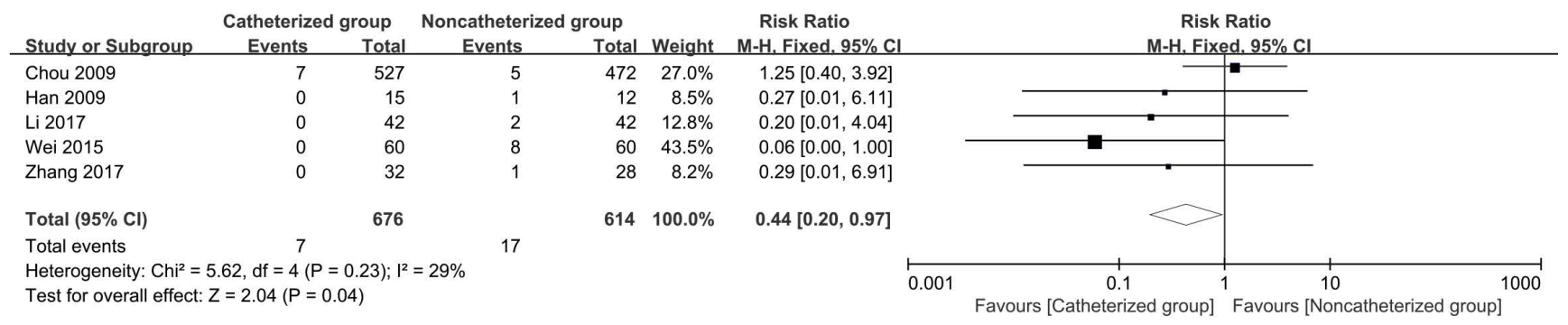

\section{b.Operative time}

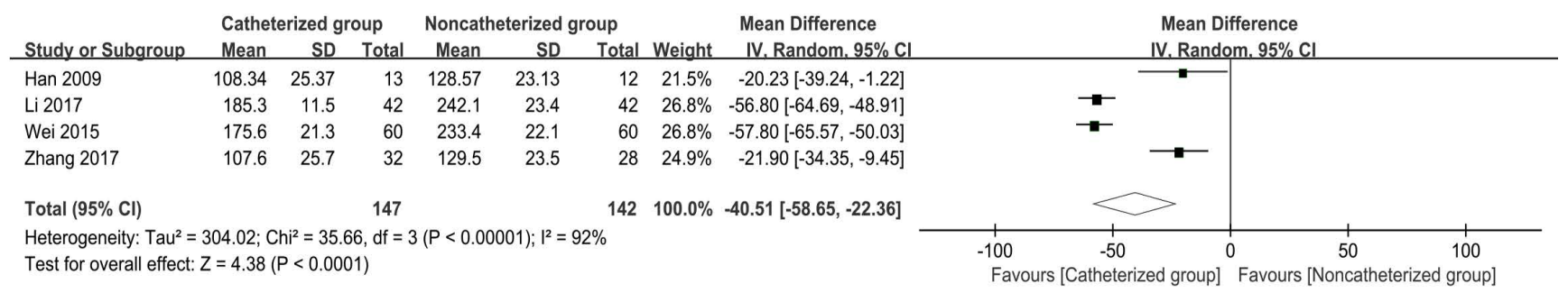

\section{c.Estimated blood loss}

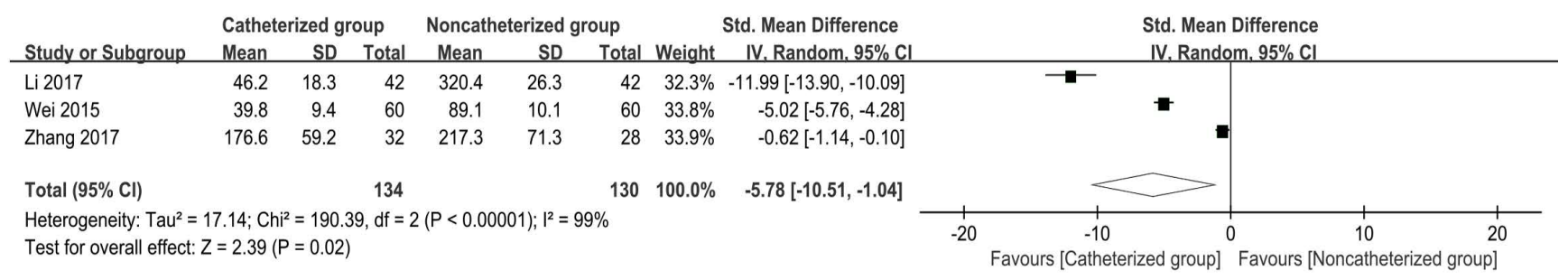

\section{d. Length of stay}

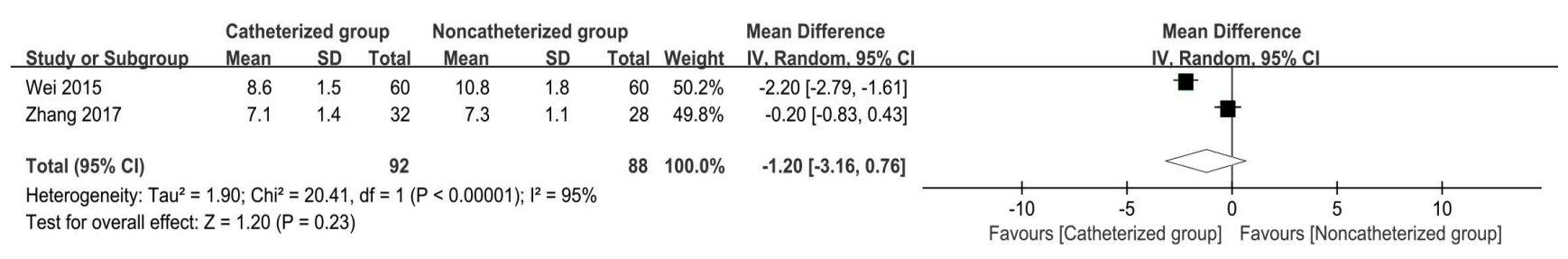

Figure 3 The outcomes of this meta-analysis.

$(2,17,19-20)$, while others are concerned about stent-related complications $(8,21,22)$. In this scenario, we attempt to explore this relationship using a meta-analysis of 5 RCTs (8-12) at home and abroad to provide an evidence to guide clinical practice.

Our study observed a statistically significant decreased risk of ureteral injury in favor of CG group. The catheters straighten the ureters and offer clear visualization through the peritoneum without invasively exploring the retroperitoneal area (2). The specific advantages of preset catheter placement were as followed (9): (I) it can make the ureter bulge, with a clearer outline and easy identification; (II) it can increase the stiffness of the ureter, and the visual sense is more obvious when the surgical instrument is 
touched; (III) it can makes the ureter and its surrounding tissues and organs more clear, which is beneficial for separation; (IV) it can detect ureteral damage promptly and reminds the surgeon to deal with it in time; (V) it can avoid blindness of operation. Thus, prophylactic ureteral catheterization may shorten operative time and reduce EBL, which is consistent with the results of this meta-analysis. All studies (8-12) included in this meta-analysis did not report major catheter-related complications, and we may consider that this simple procedure is safe. Additionally, only one study conducted by Chou and his colleagues (8) reported the severity of ureteral injuries and they found that patients in CG group had a lower severity of IUI than their counterparts.

Schimpf and his colleagues (23) performed a decisiontree analysis of clinical scenarios of using universal ureteral catheterization compared with no catheterization for benign abdominal hysterectomy and radical hysterectomy, and they found that universal ureteral catheterization is cost saving when the rate of ureteral injury during benign abdominal hysterectomy or radical hysterectomy is greater than $3.2 \%$. Four (9-12) out of five studies (8-12) included in this meta-analysis showed that the IUI rate of NCG group was greater than $3.2 \%$. Therefore, ureteral catheterization should be recommended for complicated gynecological surgery or unskilled surgeons to prevent IUI and save medical costs. Besides, lighted ureteral stents or real-time visualization of ureters using indocyanine green have been used in laparoscopic pelvic surgeries to make surgery safer $(24,25)$.

To our knowledge, our study is the first meta-analysis that evaluates laparoscopic gynecologic surgery with or without preset ureter catheter. However, our study does have the following limitations. Foremost, the broad heterogeneity in study populations, designs, and definitions of outcome measures. Secondly, the limited RCTs and the relatively small sample size make it difficult to emphatically confirm the advantages of prophylactic catheterization. Thirdly, the limitation of the study area prevented us from assessing the efficacy of preset ureteral catheter in foreign patients. Further large prospective randomized, double-blind study conducted by large volume and experienced surgeons are still needed to confirm our findings and definitively resolve this important controversy in gynecology.

\section{Conclusions}

Current evidence indicates that prophylactic ureteral catheter placement has the advantages of reducing ureteral injury, shortening the operative time and reducing the amount of bleeding. It might serve as a routine preoperative preparation choice for laparoscopic gynecological surgery, especially with pelvic adhesion. Further large volume, multicenter well-designed trials are warranted before making the final clinical guidelines.

\section{Acknowledgments}

Funding: This work was supported by the 1.3.5 Project for Disciplines of Excellence, West China Hospital, Sichuan University (ZY2016104) and Pillar Program from Department of Science and Technology of Sichuan Province (2018SZ0219). The funders had no role in study design, data collection or analysis, preparation of the manuscript, or the decision to publish.

\section{Footnote}

Reporting Checklist: The authors have completed the PRISMA reporting checklist. Available at http://dx.doi. org/10.21037/tau-20-674

Conflicts of Interest: All authors have completed the ICMJE uniform disclosure form (available at http://dx.doi. org/10.21037/tau-20-674). The authors have no conflicts of interest to declare.

Ethical Statement: The authors are accountable for all aspects of the work in ensuring that questions related to the accuracy or integrity of any part of the work are appropriately investigated and resolved.

Open Access Statement: This is an Open Access article distributed in accordance with the Creative Commons Attribution-NonCommercial-NoDerivs 4.0 International License (CC BY-NC-ND 4.0), which permits the noncommercial replication and distribution of the article with the strict proviso that no changes or edits are made and the original work is properly cited (including links to both the formal publication through the relevant DOI and the license). See: https://creativecommons.org/licenses/by-nc-nd/4.0/.

\section{References}

1. Pal DK, Wats V, Ghosh B. Urologic complications following obstetrics and gynecologicai surgery: Our 
experience in a tertiary care hospital. Urol Ann 2016;8:26-30.

2. Tanaka $\mathrm{Y}$, Asada H, Kuji N, et al. Ureteral catheter placement for prevention of ureteral injury during laparoscopic hysterectomy. J Obstet Gynaecol Res 2008;34:67-72.

3. Parpala-Spårman T, Paananen I, Santala M, et al. Increasing numbers of ureteric injuries after the introduction of laparoscopic surgery. Scand J Urol Nephrol 2008;42:422-7.

4. Selzman AA, Spirnak JP. Iatrogenic ureteral injuries: a 20-year experience in treating 165 injuries. J Urol 1996;155:878-81.

5. Elliott SP, McAninch JW. Ureteral injuries: external and iatrogenic. Urol Clin North Am 2006;33:55-66.

6. Moher D, Liberati A, Tetzlaff J, et al. Preferred reporting items for systematic reviews and meta-analyses: the PRISMA statement. PLoS Med 2009;6:e1000097.

7. Oxford Centre for evidence-based medicine: levels of evidence 2009. Available online: http://www.cebm.net/ oxford-centreevidence-based-medicine-levels-evidencemarch-2009/

8. Chou MT, Wang CJ, Lien RC. Prophylactic ureteral catheterization in gynecologic surgery: a 12-year randomized trial in a community hospital. Int Urogynecol J Pelvic Floor Dysfunct 2009;20:689-93.

9. Zhang N, Qiang Y, Zhai Z, et al. Randomized controlled study of ureteral catheter placement for prevention of injury during laparoscopic gynaecologic surgery. Biomed Res 2017;28:3059-62.

10. Han F, Tang S, Niu G, et al. Ureteral catheter placement for prevention of ureteral injury during laparoscopy in gynecology. Chin J Endourology (Electronic Edition) 2009;3:493-6.

11. Wei Z, Guo F, Gao Y, et al. Application of ureteral catheter in the prevention of ureteral injury under gynecologic laparoscopic surgery. China Health Standard Management 2015;6:26-7.

12. Li D, Li D, Jia L, et al. Clinical application and discussion of ureteral catheter in gynecological laparoscopic surgery to prevent ureteral injury. Contemporary Medicine 2017;23:48-9.

13. Higgins JP, Thompson SG, Deeks JJ, et al. Measuring inconsistency in meta-analyses. BMJ 2003;327:557-60.

14. Higgins JP, Thompson SG. Quantifying heterogeneity in a meta-analysis. Stat Med 2002;21:1539-58.
15. Liguori G, Dobrinja C, Pavan N, et al. Iatrogenic ureteral injury during laparoscopic colectomy: incidence and prevention A current literature review. Ann Ital Chir 2016;87:446-55.

16. Khan F, Kenton K. Intraoperative Injury in Reconstructive Pelvic Surgery. J Pelvic Med Surg 2006;12:241-56.

17. Han L, Cao R, Jiang JY, et al. Preset ureter catheter in laparoscopic radical hysterectomy of cervical cancer. Genet Mol Res 2014;13:3638-45.

18. Teeluckdharry B, Gilmour D, Flowerdew G. Urinary Tract Injury at Benign Gynecologic Surgery and the Role of Cystoscopy: A Systematic Review and Meta-analysis. Obstet Gynecol 2015;126:1161-9.

19. Koh LW, Koh PH, Lin LC, et al. A simple procedure for the prevention of ureteral injury in laparoscopic-assisted vaginal hysterectomy. J Am Assoc Gynecol Laparosc 2004;11:167-9.

20. Quinlan DJ, Townsend DE, Johnson GH. Are ureteral catheters in gynecologic surgery beneficial or hazardous? J Am Assoc Gynecol Laparosc 1995;3:61-5.

21. Kuno K, Menzin A, Kauder HH, et al. Prophylactic ureteral catheterization in gynecologic surgery. J Urol 1999;162:1563.

22. Wood EC, Maher P, Pelosi MA. Routine use of ureteric catheters at laparoscopic hysterectomy may cause unnecessary complications. J Am Assoc Gynecol Laparosc 1996;3:393-7.

23. Schimpf MO, Gottenger EE, Wagner JR. Universal ureteral stent placement at hysterectomy to identify ureteral injury: a decision analysis. BJOG 2008;115:1151-8.

24. Mandovra P, Kalikar V, Patankar RV. Real-time visualization of ureters using indocyanine green during laparoscopic surgeries: can we make surgery safer? Surg Innov 2019;26:464-8.

25. Boyan WP Jr, Lavy D, Dinallo A, et al. Lighted ureteral stents in laparoscopic colorectal surgery;a five-year experience. Ann Transl Med 2017;5:44.

Cite this article as: Feng D, Tang Y, Yang Y, Wei X, Han P, Wei W. Does prophylactic ureteral catheter placement offer any advantage for laparoscopic gynecological surgery? A urologist' perspective from a systematic review and meta-analysis. Trans Androl Urol 2020;9(5):2262-2269. doi:10.21037/tau-20-674 